\title{
CORONAVIRUS JHM OMP1 PATHOGENESIS IN \\ OWL MONKEY CNS AND CORONAVIRUS INFECTION \\ OF OWL MONKEY CNS VIA PERIPHERAL ROUTES
}

\author{
Gary F. Cabirac ${ }^{1,2}$, Kenneth F. Soike ${ }^{3}$, Catalin Butunoi ${ }^{1}$, Kristen Hoel ${ }^{1}$, \\ Steven Johnson ${ }^{1}$, Guang-Yun Cai ${ }^{1}$, and Ronald S. Murray ${ }^{1}$ \\ ${ }^{1}$ Rocky Mountain Multiple Sclerosis Center, Colorado Neurological Institute \\ and Swedish Medical Center, Englewood, CO 80150 \\ ${ }^{2}$ Department of Biochemistry, Biophysics and Genetics, University of \\ Colorado Health Sciences Center, Denver, CO 80262 \\ ${ }^{3}$ Department of Microbiology, Tulane Regional Primate Research Center \\ Covington, LA 70433
}

\begin{abstract}
Two separate studies are described in this report. First, 5 Owl monkeys were inoculated intracerebrally (IC) with coronavirus JHM OMP1; this virus isolate was cultured from the brain of an animal inoculated with uncloned MHV JHM. Two of the animals became neurological impaired and were sacrificed; these animals had developed severe encephalomyelitis as previously described ${ }^{1}$. Two of the remaining 3 healthy animals were inoculated IC again at 90 days post-inoculation (DPI) and all 3 were sacrificed approximately 5 months after the first virus inoculation. Despite the lack of detectable infectious virus, viral RNA and antigen, all 3 animals had significant white matter inflammation and areas of demyelination in the spinal cord. In the second study 4 Owl monkeys were inoculated intranasally (IN) and ocularly and 4 inoculated intravenously (IV) with JHM OMP1. The animals were sacrificed between 16 and 215 DPI with 2 IN and 2 IV animals receiving a second IV inoculum at 152 DPI. Viral RNA and/or antigen was detected in the brains of all animals and the distribution corresponded to areas of inflammation and edema. One of the animals that received the second inoculum developed neurological impairment and subsequent analysis of tissues showed viral antigen in both brain and spinal cord. Viral products were predominantly found in blood vessels suggesting hematogenous spread with entry into the central nervous system (CNS) through endothelium.
\end{abstract}




\section{INTRODUCTION}

In humans, coronaviruses are a leading upper respiratory tract pathogen ${ }^{2}$ and are associated with gastroenteritis ${ }^{3}$ but have never been proven to cause serious human illness. However, we have reported that coronavirus RNA and antigen are detectable in demyelinating lesions of human multiple sclerosis brain ${ }^{4}$ and that, following IC inoculation, coronaviruses can productively infect and disseminate in the brains of primates causing encephalomyelitis and demyelination ${ }^{1}$. In addition, two recent reports demonstrate that human coronavirus (HCV) 229E RNA can be detected in multiple sclerosis brain by the polymerase chain reaction (PCR) assay ${ }^{5}$ and that the cell surface glycoprotein aminopeptidase $\mathrm{N}$, a polypeptide expressed on nerve synapses, functions as a receptor for $229 \mathrm{E}^{6}$. Collectively these data suggest that coronaviruses are capable of natural infection of the human central nervous system (CNS) and hence may be involved in neurologic disease.

In an effort to further define the interaction of coronaviruses with human CNS tissue, we have utilized primates to investigate the disease potential of these viruses. The first part of this report will describe results of IC inoculation of JHM OMP1 into Owl monkeys. Data on two of the animals that developed neurological impairment have already been published $^{1}$; presented here are results from the remaining animals that were sacrificed at later times. Secondly, we demonstrate that JHM OMP1 can gain access to primate CNS following IN or IV inoculation.

\section{METHODS AND RESULTS}

\section{IC Inoculated Animals}

Five outbred young adult Owl monkeys were inoculated IC as previously described ${ }^{1}$ with JHM OMP1. Assays for infectious virus and neutralizing antibody, in situ hybridization, immunohistochemical and histochemical staining were performed as described $^{1}$. Two of the five animals, K191 and K063, were observed with apparent neurological dysfunction on 10 and 12 DPI, respectively, and sacrificed. The results of analysis of tissue from these two animals has previously been described ${ }^{1}$. Briefly, infectious virus was cultured from the brains of both animals and from the blood of K063, abundant amounts of viral RNA and antigen were detected in both grey and white matter cells and pathologic examination of tissues revealed that both animals had developed severe encephalomyelitis. The remaining 3 animals, K189, K171 and K072, appeared normal and by 90 DPI had no detectable neutralizing antibody. Two of these animals, K189 and K171, were inoculated IC with approximately $5 \times 10^{4}$ TCID50 of JHM OMP1 at this time. Both animals seroconverted after this second inoculation developing low titers of 1:20 and 1:10 for K189 and K171, respectively. These two animals and K072 were sacrificed 150 days after the initial inoculation; all three animals appeared healthy at the time of sacrifice.

Infectious virus could not be cultured from brain, CSF or blood obtained from these three animals. Histochemical staining of tissues revealed that all three animals had white matter inflammation, demyelination in the spinal cord and meningitis. However, no virus products could be detected in these affected areas by in situ hybridization or immunohistochemical staining methods. PCR amplification of RNA extracted from tissue sections failed to produce coronavirus specific products. One of the most striking observation was that all three animals had defined areas of multilevel demyelination in the dorsolateral spinal cord. Figure 1A shows a luxol-fast blue/periodic acid-Schiff stain of a section from K189 demonstrating the area of demyelination. Interestingly, these regions of spinal cord demyelination were accompanied by gliosis. Staining with anti-glial 
fibrillary acidic protein (GFAP) monoclonal (Fig. 1B) showed the predominant cell type to be reactive astrocytes. Immunostaining for macrophages/monocytes was negative in these regions (data not shown).

\section{Peripherally Infected Animals}

Four Owl monkeys were inoculated intravenously (IV) and four intranasally (IN) plus ocularly with coronavirus JHM OMP1. The four IV inoculated animals were injected with $1 \mathrm{ml}$ of a $1 \times 10^{6} \mathrm{TCID} 50 / \mathrm{ml}$ titer inoculum in the saphenous vein. The other four animals received $1 \mathrm{ml}$ of the same virus inoculum divided between two nostrils plus 2 drops of this inoculum in the right eye following corneal scarification. Preparation of the virus inoculum has been described ${ }^{1}$. Animals were sacrificed at the indicated time points then tissue from brain and spinal cord was screened for areas of pathology by histochemical staining. Those tissues containing regions of pathology, unaffected tissues and tissue from a sham inoculated control animal were processed for the detection of viral RNA and antigen. In situ hybridization analysis was performed as described above but a modification of our immunostaining procedure was used on tissues from these peripherally inoculated animals. The modifications are as follows. Formalin-fixed, paraffin embedded tissue was sectioned, de-paraffinized and incubated in $\mathrm{Ca}^{+2} / \mathrm{Mg}^{+2}$-free PBS containing $0.25 \%$ trypsin at room temperature for 90 minutes then washed three times in PBS. Incubation of the primary antibody was done at $4{ }^{\circ} \mathrm{C}$ overnight. Secondary antibody was biotinylated rabbit anti-mouse immunoglobulins (Dako), incubated at room temperature for 30 minutes. Following antibody treatment, tissue was incubated for 20 minutes at room temperature with alkaline phosphatase conjugated strepavidin (BioGenex), washed and then the chromogen Fast Red was incubated for 20 minutes at $37^{\circ} \mathrm{C}$.

Two animals, K183 and K172, inoculated IV and IN respectively, were sacrificed 16 DPI. Throat swab and blood cultures from K183 and throat, blood and conjunctiva/corneal swab cultures from K172 taken on 1, 2, 5, 7, and 9 DPI were negative for infectious virus. Sera from both animals collected 14 DPI did not have detectable neutralizing antibody. CSF and tissue from brain, spinal cord and lung were negative for infectious virus. Histochemical staining revealed varying degrees of meningitis, ependymitis, and mild white matter edema and inflammation in both animals and choroiditis in K183. Viral RNA was not detectable by in situ hybridization in K172 but evident in a small percentage of blood vessel endothelium in the brain of the IV inoculated animal K183. Viral antigen was detectable with the nucleocapsid specific monoclonal antibody J.3.1 ${ }^{7}$ in brain tissue from both of these animals. Positive staining for viral antigen was observed predominantly in blood vessel walls; occasionally, cells surrounding positive vessels appeared to contain viral antigen. Viral products were not detected in trigeminal ganglia or olafactory blubs from IN inoculated animal K172.

Animals K186 and K192, IV and IN inoculated respectively, were sacrificed 35 DPI. A throat culture obtained 1 DPI from K192 was positive for infectious virus but all other throat, blood and conjunctive (K192) cultures from both animals were negative. At the time of sacrifice, brain and spinal cord did not contain infectious virus. As with the two animals sacrificed 16 DPI, K186 and K192 never produced detectable neutralizing antibody. Meningitis, ependymitis, choroiditis and mild white matter edema and inflammation were evident in both K186 and K192. In situ hybridization (Fig. 1C) and immunohistochemical staining revealed viral RNA and antigen in blood vessel endothelium and adjacent areas in both of these animals. It appeared that viral products were more widely distributed in the IV inoculated animal, K186. As with K172, viral RNA or antigen were not detected in trigeminal ganglia and olafactory blubs from K192.

Following the analysis of tissue from the first 4 animals, the remaining 4 animals, K181 (IV), K179 (IV), K187 (IN) and K194 (IN), were inoculated with a second dose of 
virus. $150 \mathrm{DPI}$ after receiving the first inoculum, these animals were inoculated IV with the second inoculum. Assays of neutralizing antibody in sera from these animals showed that these 4 animals had seroconverted before receiving the second dose of virus; the highest titer was 1:80 for IN inoculated animal K187 (35 DPI) and the lowest was 1:10 for IV animal K179 (35 DPI). To varying degrees, the second inoculum caused a rise in titers; 1:320 for K187 at 183 DPI and 1:20 for K181 at 183 DPI and K194 at 215 DPI are the maximum and minimum titers recorded. Blood, throat and conjunctival cultures taken on 1,2,5, 7, and 9 DPI (first inoculation) were negative for infectious virus; no additional cultures were taken after the second virus inoculum. On 189 DPI, 36 days after administration of the second dose of virus, animal K179 was observed with hind limb weakness and tremors. This animal was sacrificed 194 DPI and tissue processed as before. Histochemical staining showed that this animal had detectable viral antigen not only in the brain but also in the spinal cord. Again, most of the detected antigen was associated with blood vessels but occasionally, surrounding areas stained positive for nucleocapsid protein. The remaining 3 animals, K181, K187 and K194 were sacrificed 215 DPI (62 days after second inoculation). Immunohistochemical analysis of tissue from these animals revealed the same results as described above, i.e., viral antigen in vessels and surrounding regions. Quantitatively, there appeared to be much less antigen in animal K187 compared to K181 and K194 and analyzed tissue from these three animals showed less antigen when compared to the animal with neurological dysfunction, K179.
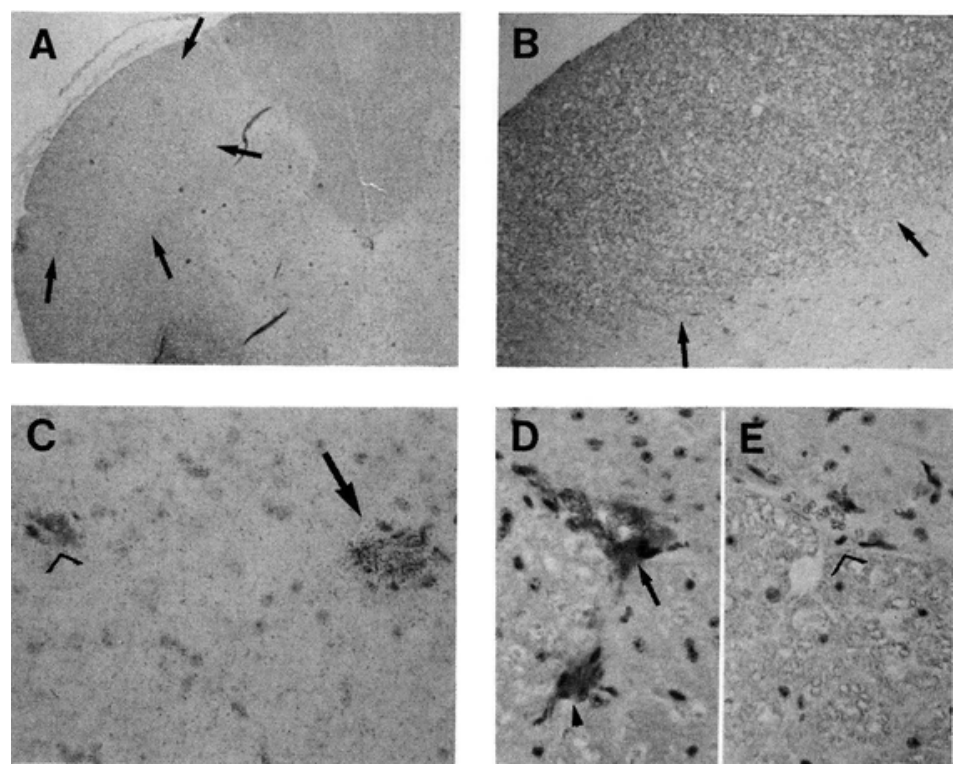

Figure 1. Histochemical and immunohistochemical staining of spinal cord from IC inoculated monkey and in situ hybridization and viral antigen staining of tissue from peripherally inoculated animals. A Luxol-fast blue/periodic acid-Schiff staining of myelin in spinal cord from K189. Area of decreased staining shown by arrows indicates demyelination. B - An adjacent section to that shown in A was immunostained with monoclonal against GFAP. Arrows show region of staining indicating extensive astrocyte proliferation in demyelinated area. C - In situ hybridization of tissue from IN inoculated animal K192 showing higher density of grains over vessel (arrow); adjacent to this vessel is another vessel (open arrowhead) that has only background levels of grain development. D - Positive nucleocapsid staining with monoclonal J.3.1 in section from animal K179. Arrow shows staining in vessel and arrowhead show staining in surrounding areas. E -Adjacent section to that shown in D in which the primary antibody was normal mouse serum. Note lack of staining in vessel (open arrowhead). 


\section{DISCUSSION}

The data described in this report in addition to the previously published study of active coronavirus infection in primate CNS demonstrates the value of primates as a model system. It is now evident that coronaviruses can replicate within primate CNS and produce disease. Results presented here extend the earlier work and show that the inflammation and demyelination induced by coronavirus infection persists in the absence of detectable virus and, perhaps more importantly, that a coronavirus can gain access to primate CNS following peripheral infection.

Coronavirus induced demyelination in the rodent system is well documented ${ }^{8-18}$. Therefore the failure to detect virus in areas of demyelination and inflammation in the IC infected primates was not unexpected. However, at this time we do not know if this aspect of primate CNS disease is unique to JHM OMP1. We state this since in our original report $^{1}$ all of the animals infected with JHM, the putative MS isolate SD and JHM OMP1 were sacrificed no more than 120 days post-inoculation and all but one of these animals had detectable levels of virus or viral products in the affected areas. The animals described here were sacrificed 150 DPI and therefore pathology in the absence of virus may be the result of longer infection times. While we could not detect viral RNA by PCR in these affected areas, low levels of RNA may be present. In our hands the efficiency of PCR amplification of RNA extracted from formalin fixed tissue is low compared to that done on RNA from snap-frozen or fresh samples. However, with this caveat in mind, the possibility that coronavirus infection in these primates triggered an autoimmune reaction needs to be investigated. Obviously a study in which infected primates are followed for a much longer time course needs to be done. If it can be determined that these viruses are completely cleared from the CNS but that an autoimmune response has been initiated then the primates would serve as an excellent model for investigating coronavirus induced autoimmune demyelination in humans.

The results from the peripherally infected animals reinforces data indicating that coronaviruses are CNS pathogens in humans. The data suggests that for both the IV and IN inoculated animals, virus entered the CNS through blood vessel endothelium as opposed to neural routes. The antibody data indicates that virus replication did occur in these animals. While some assays for infectious virus were performed, more rigorous tests are needed to determine the primary sites of infection in these animals. The one positive throat swab culture from IN inoculated animal K192 suggests that respiratory epithelium may be a reservoir for virus. Some positive antigen staining was seen in liver from two of the IV inoculated animals but it was rare and hard to repeat. While all blood sample cultures from these animals were negative for infectious virus, we need to investigate the possibility that certain populations of peripheral blood cells can be infected. This is emphasized by the fact that one of the earlier IC inoculated animals (K063) had detectable virus in the blood. Interestingly, there seemed to be a correlation between neutralizing antibody titers and amounts of viral antigen detected in these peripherally infected animals, i.e., those animals with poor antibody response had higher amounts of viral antigen while animals; in particular IN animal K187, with higher titers had lower antigen levels. In this context it is important to note that the animal that developed neurological impairment, $\mathrm{K} 179$, had the lowest titer of all four re-inoculated animals. It will be necessary to determine the relationship between the cellular plus humoral immunity against coronaviruses and CNS disease produced by multiple peripheral infections. While other animal species are excellent for investigating coronavirus induced CNS disease these studies demonstrate the usefulness of primates as a model system and reinforce previous data implicating coronaviruses as human CNS pathogens. 


\section{REFERENCES}

1. R.S.Murray, G-Y Cai, K.Hoel, et al, Virology 188:274 (1992).

2. T.Hovi, H.Kainulainen, B.Ziola, A.Salmi, Med.Virol. 3:313 (1979).

3. S.Resta, J.P.Luby, C.R.Rosenfeld, J.D.Siegel, Science 229:978 (1985).

4. R.S.Murray, B.Brown, D.A.Brian, G.F.Cabirac, Ann.Neurol. $31: 525$ (1992).

5. J.N.Stewart, S.Mounir, P.J.Talbot, Virology. In Press (1992).

6. $\quad$ C.L.Yeager, R.A.Ashmun, R.K.Williams, et al, Nature 357:420 (1992).

7. J.O.Fleming, F.A.K.El-Zaatari, W.Gilmore, et al, Arch.Neurol. 45:629 (1988).

8. O.T.Bailey, A.M.Pappenheimer, F.S.Cheever, J.B.Daniels, J.Exp.Med. 90:195 (1949).

9. A.M. Pappenheimer, J.Natl.Can.Inst. 20:879 (1958).

10. L.P.Weiner, Arch.Neurol. 28:298 (1973).

11. R.M.Herndon, D.E.Griffin, U.McCormick, L.P.Weiner, Arch.Neurol. $32: 32$ (1975).

12. A.Lucas, W.Flintoff, R.Anderson, et al, Cell 12:553 (1977).

13. K.Nagashima, H.Wege, et al, Acta.Neuropathol. 44:63 (1978).

14. O.Sorensen, D.Percy, S.Dales, Arch.Neurol. 37:478 (1980).

15. S.A.Stohlman, L.P.Weiner, Neurology 31:38 (1981).

16. R.Watanabe, H.Wege, V.Ter Meulen, Nature 305:150 (1983).

17. E.Lavi, D.H.Gilden, M.K.Highkin, S.R.Weiss, J.Virol. 51:563 (1984).

18. S.Kyuwa, K.Yamaguchi, Toyoda, K.Fujiwara, J.Virol. 65:1789 (1991). 